\title{
PACIENTES COM PSORÍASE: ADAPTAÇÃO PSICOSSOCIAL E CARACTERÍSTICAS DE PERSONALIDADE
}

\author{
PATIENTS WITH PSORIASIS: PSYCHOSOCIAL ADAPTATION AND PERSONALITY FEATURES
}

Regina C. Mingorance ${ }^{1}$, Sonia R. Loureiro², Liyoko Okino ${ }^{3} \&$ Norma T. Foss ${ }^{4}$

\begin{abstract}
'Docente da Universidade de Ribeirão Preto - UNAERP. 'Docente do Departamento de Neurologia, Psiquiatria e Psicologia Médica, ${ }^{3}$ Médica Dermatologista e Colaboradora do Departamento de Dermatologia, ${ }^{4}$ Docente do Departamento de Dermatologia; Faculdade de Medicina de Ribeirão Preto - USP.

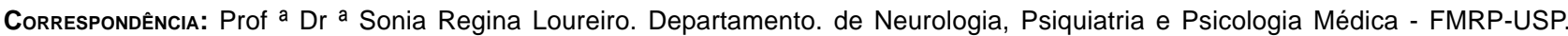
Av. 9 de julho, 980 - Ribeirão Preto, SP. CEP: 14025-000 Fone: (016) 6250309 Fax: (016) 6350713
\end{abstract}

MINGORANCE RC; LOUREIRO SR; OKINO L \& FOSS NT. Pacientes com psoríase: adaptação psicossocial e características de personalidade. Medicina, Ribeirão Preto, 34: 315-324, jul./dez. 2001.

RESUMO: A psoríase tem suscitado interesse na investigação por causa dos aspectos psicológicos e sociais de seus portadores. Objetivou-se avaliar as condições psicológicas e adaptativas de pacientes com psoríase e suas relações com variáveis demográficas e condições clínicas. Foram avaliados, no Ambulatório de Dermatologia do Hospital das Clínicas da FMRP - USP, 25 mulheres e 25 homens com idade entre 20-50 anos com diagnóstico de psoríase vulgar. Procedeu-se, em sessões individuais, à aplicação individual do Inventário Simplificado de Personalidade (ISP) e do Inventário de Qualidade de Vida (PDI), conforme as recomendações técnicas, e como complemento houve uma entrevista semi-estruturada. Os protocolos foram cotados e, comparados aos dados normativos, procedeu-se à quantificação e tratamento estatístico. Observou-se que $80 \%$ dos pacientes apresentaram tendência a desvio de traço de personalidade, predominando as escalas de Extroversão, Insanidade e Neuroticidade. A maioria relatou dificuldades de adaptação psicossocial, principalmente nas áreas de atividades rotineiras $(p<0.001)$; Extensão psoriática, faixa etária entre 20 a 30 anos, insatisfação quanto à aparência física e desvios de Insanidade e Neuroticidade mostraram-se associados a prejuízos nas atividades rotineiras, relações pessoais e lazer. Os dados apontam a inter-relação de aspectos psíquicos, adaptação psicossocial e peculiaridades clínicas da doença.

UNITERMOS: Psoríase. Qualidade de Vida. Psicologia. Inventário de Personalidade.

\section{INTRODUÇÃO}

Atualmente, é crescente o interesse pelos aspectos psicoadaptativos, envolvidos no quadro clínico da psoríase, focalizando situações de discriminação e consequiências na qualidade de vida do paciente ${ }^{(1 / 8)}$.

O funcionamento mental do paciente com psoríase tem sido associado a correlatos psíquicos, como o impacto emocional da doença; a associação do aumento de preocupações e ansiedades à piora da lesão; a presença de distúrbios no ambiente familiar; a presença de indicadores de instabilidade emocional, insegurança, alto grau de neuroticidade, caráter esquizóide e de inibição da agressividade, resultando em depressão ${ }^{(9)}$. Alto nível de depressão apresentou-se em trabalho com pacientes com psoríase associada à alta porcentagem de disseminação das lesões, além de vivências constantes de ansiedade ${ }^{(6)}$. Tais indicadores são sugestivos das dificuldades emocionais e de possível precariedade quanto ao funcionamento adaptativo. 
Em um estudo clínico, objetivando avaliar as dimensões de experiências estigmatizadoras do paciente com psoríase, foram identificados problemas relativos à auto-estima, isolamento social, rejeição, e diferenças quanto à idade, tempo de doença e gênero. Observou-se que pacientes mais velhos, pacientes do sexo feminino e pacientes com longa história de doença referiram menos sentimentos de estigmatização ${ }^{(5)}$. Foi relatada a presença de maior sentimento de culpa entre as mulheres com psoríase do que entre os homens e maior suscetibilidade às vivências de estigmatização entre as pessoas que desenvolveram a doença quando muito jovens. ${ }^{(8)}$

Apesar do alto impacto psicossocial, decorrente do desenvolvimento da doença, a intensidade do prejuízo adaptativo pode variar de acordo com a percepção do paciente ${ }^{(10)}$. Neste sentido, as condições psíquicas do paciente com psoríase podem desempenhar um papel importante na autopercepção quanto à condição física e na mediação da intensidade do impacto da doença no cotidiano.

Assim, o presente trabalho objetiva avaliar, através de inventários, os recursos adaptativos, as condições psicológicas de pacientes com psoríase e suas relações com variáveis demográficas e condições clínicas.

\section{MATERIAL E MÉTODOS}

\section{Sujeitos}

O presente estudo foi avaliado e aprovado pela Comissão de Ética em Pesquisa do HC da FMRPUSP e, atendendo às recomendações da comissão, a participação dos pacientes foi voluntária e com consentimento formal.

Foram avaliados 50 pacientes adultos, em seguimento no Ambulatório de Dermatologia do HC da FMRP-USP durante o segundo semestre de 1997, 25 do sexo masculino e 25 feminino, com diagnóstico clínico de psoríase vulgar. A idade variou entre 20 a 50 anos, sendo $24 \%$ com idade entre $20-30$ anos, $26 \%$ entre 31-40 anos e 50\% acima de 41 anos. A maioria dos pacientes $(72 \%)$ apresentou lesões localizadas, depois vieram os pacientes com lesões disseminadas (28\%). No presente trabalho, o grau de acometimento das lesões foi avaliado apenas em termos de extensão localizada ou disseminada.

\section{Instrumentos}

Para a coleta de dados, foram utilizados os procedimentos relatados a seguir. a) Entrevista semi-estruturada, elaborada em função dos objetivos do trabalho, abordando: 1) dados de identificação; 2) questões relativas ao estado geral de saúde e ao acompanhamento médico; 3) aspectos relativos à adaptação social, abrangendo as percepções do paciente frente ao quadro clínico, dermatológico, à aparência física, os relacionamentos, às atividades ocupacionais e de lazer e ao impacto da doença na vida cotidiana.

b) Inventário Simplificado de Personalidade (ISP) ${ }^{(11)}$, escolhido por ser um instrumento adaptado ao contexto brasileiro e utilizado na avaliação de pacientes com patologias orgânicas, se portadores de úlcera péptica duodenal ${ }^{(12)}$. O ISP permite detectar pessoas que potencialmente apresentem distúrbios psiquiátricos, possibilitando discriminar cinco setores específicos de personalidade: Insanidade, Comportamento Anti-Social, Neuroticidade, Extroversão e Dissimulação. Constitui-se de 130 afirmativas com opções falso e verdadeiro, sendo solicitada a escolha de uma delas. Cada setor avaliado compreende um conjunto de sinais e sintomas de manifestações psíquicas e comportamentais com características comuns. A Insanidade, caracteriza-se pela presença de indicadores de isolamento, desconfiança, ansiedade paranóide, perda de controle, distorções sensoriais e sensações de estranheza ou de irrealidade. A escala Comportamento Anti-social compreende indicadores de características sociopáticas. A escala Neuroticidade, reúne indicadores relativos à ansiedade e à adaptação social. A escala Extroversão avalia a habilidade e os níveis de controle sobre a impulsividade, compreendendo desde o controle rígido até as manifestações francas. A escala Dissimulação apresenta-se como um elemento de correção, avaliando o grau de defesa da pessoa com relação ao teste.

c) Inventário de Qualidade de Vida - Psoriasis Disability Index (PDI) ${ }^{(1,2,3,13)}$. O instrumento propõe a avaliação da qualidade de vida de pessoas com psoríase, através de indicadores do impacto da doença sobre o estilo de vida e o cotidiano em áreas específicas. Compreende 15 questões de múltipla escolha, sendo oito referentes a atividades rotineiras e hábitos, duas às relações interpessoais e cinco ao lazer. Cada questão apresenta quatro alternativas que se diferenciam pelo grau de intensidade do impacto da doença no cotidiano. 
A utilização do PDI, no presente trabalho, exigiu sua tradução e adaptação. Para tal, procedeu-se à aplicação do inventário traduzido em 10 pacientes com psoríase. Após a aplicação, foram solicitados comentários quanto à compreensão das questões, com posterior adequação do instrumento. Com base nas sugestões, o instrumento foi reformulado e procedeu-se novamente à comparação com o original, em Inglês, visando verificar a preservação do sentido original na formulação das questões traduzidas.

\section{Coleta dos dados}

A coleta de dados foi realizada no Ambulatório de Dermatologia da FMRP-USP, pela primeira autora, psicóloga, em sessões individuais de aproximadamente uma hora e trinta minutos, resguardando-se a privacidade dos pacientes. Procedeu-se a um breve rapport seguido da entrevista e da aplicação do PDI e ISP, conforme recomendações técnicas.

\section{Análise dos dados}

Os dados obtidos através da análise das entrevistas foram categorizados, visando à caracterização do quadro geral dos pacientes.

$\mathrm{Na}$ análise do ISP, a cotação foi realizada através de máscaras apropriadas para a quantificação de cada subescala, procedendo-se à comparação com os dados normativos ${ }^{(12)}$.

Quanto ao PDI, para cada questão, as alternativas receberam escores variando de zero a três, de acordo com o grau de prejuízo, sendo o escore zero referente à ausência de prejuízo. A pontuação variou do mínimo - zero - à pontuação máxima de 45 pontos, a qual indicou alto índice de prejuízo na adaptação social, em função da doença.

Os dados foram quantificados sob a forma de porcentagem e valores médios. Para a comparação dos dados obtidos através das entrevistas, do PDI e do ISP foram utilizados os testes de Kruskal-Wallis e Mann-Whitney.

\section{RESULTADOS}

Na Tabela I, são apresentadas as comparações dos dados, através do teste de Kuskall-Wallys, referentes ao impacto da doença na qualidade de vida dos pacientes avaliados através do PDI.
No que se refere ao impacto causado pela psoríase nas áreas específicas, avaliadas pelo PDI - atividades rotineiras, relações pessoais e lazer - os pacientes apresentaram prejuízo significativamente maior nas atividades rotineiras, quando comparado no das relações pessoais $(\mathrm{p}<0.01)$ e ao do lazer $(\mathrm{p}<0.01)$.

$\mathrm{Na}$ Tabela II, são apresentadas as comparações dos dados, através do teste de Kuskall-Wallys, estatisticamente significantes, entre as áreas referentes à qualidade de vida, avaliadas pelo PDI e às variáveis, faixa etária e extensão das lesões.

Em relação à extensão das lesões, os pacientes com lesões disseminadas referiram prejuízo significativamente maior em suas atividades rotineiras $(\mathrm{p}<0.05)$, lazer $(p<0.07)$ e qualidade de vida geral $(\mathrm{p}<0.05)$, compreendendo as três áreas avaliadas, em comparação aos que apresentaram lesões localizadas. A variável idade também apresentou diferenças significativas quanto aos relacionamentos pessoais. Pacientes com idade entre 20-30 anos apresentaram um prejuízo significativamente maior nos seus relacionamentos pessoais em comparação aos pacientes com idade entre $31-40$ anos $(\mathrm{p}<0.05)$.

Na Tabela III, são apresentados os dados relativos aos traços de personalidade avaliados (ISP) e à direção dos desvios.

Dos pacientes avaliados, $80 \%$ apresentaram tendência a desvio, em, pelo menos, um tipo de traço de personalidade, predominando as escalas Extroversão, Insanidade, e Neuroticidade. Na escala Extroversão, $18 \%$ apresentaram alto escore, indicando indícios de descontrole emocional, impulsividade, exibicionismo, e $18 \%$ apresentaram baixo escore, indicativo de supercontrole emocional e atitude passiva frente às situações. Na escala Insanidade, $28 \%$ dos pacientes apresentaram alto escore, sugerindo possíveis distúrbios psiquiátricos, e $6 \%$ apresentaram
Tabela I: Média, desvio-padrão e mediana das respostas fornecidas pelos pacientes, em relação à influência da psoríase na qualidade de vida, avaliada pelo PDI.

\begin{tabular}{|lcccc|}
\hline Áreas do PDI & Média & Desvio-padrão & Mediana & Comparações \\
\hline 1. Atividades rotineiras & 0.27 & 0.2 & 0.21 & $\begin{array}{l}1>2^{* *} \\
1>3^{* *}\end{array}$ \\
2. Relações pessoais & 0.18 & 0.3 & 0.17 & \\
3. Lazer & 0.17 & 0.2 & 0.13 & \\
\hline${ }^{* *} \mathrm{P}<0.01$ & & & & \\
\hline
\end{tabular}




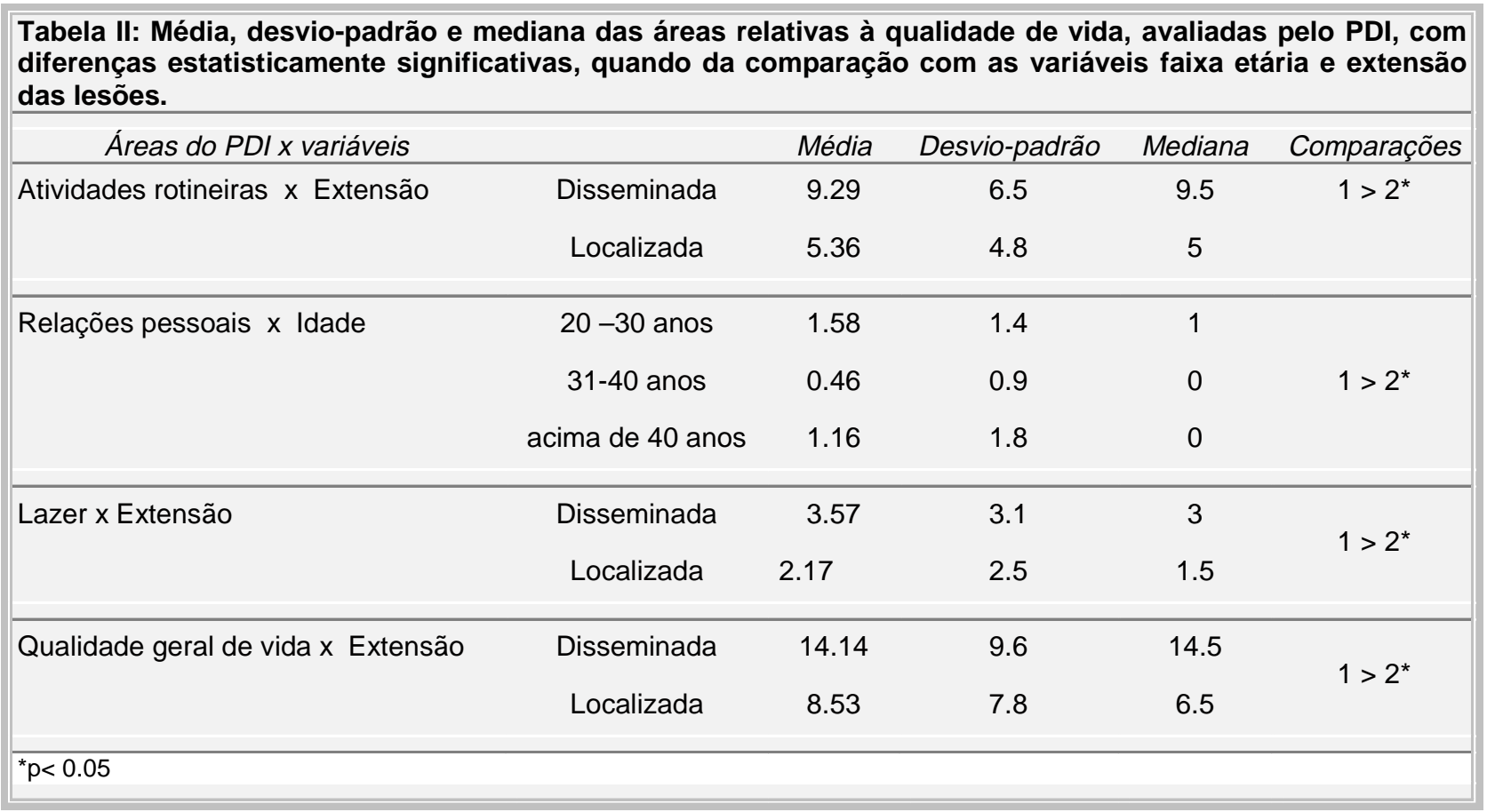

baixo escore de desvio, indicando a presença de sentimentos de isolamento, ansiedade paranóide e dificuldade na contenção dos impulsos. Quanto à escala Neuroticidade, $22 \%$ dos pacientes apresentaram alto escore, próprio de rigidez e alta suscetibilidade à ansiedade, associado a reações neuróticas, mediante situações estressantes, com manifestações somáticas, psíquicas e de desadaptação social e 10\% apresentaram baixo escore, indicando sentimentos de satisfação pessoal, flexibilidade a novas situações e à adaptação social.

A Tabela IV apresenta as comparações dos dados, através do teste de Kuskall-Wallys, entre presença e ausência de desvio nas escalas Insanidade, Anti-Social e Neuroticidade (ISP) e prejuízo nas áreas de atividades rotineiras, relações pessoais, lazer e qualidade geral de vida (PDI).

$\mathrm{Na}$ área específica de atividades rotineiras, quando da comparação com desvios de traços de personalidade, observou-se uma diferença estatisticamente significativa nas escalas Insanidade $(\mathrm{p}<0.01)$, AntiSocial $(\mathrm{p}<0.05)$ e Neuroticidade $(\mathrm{p}<0.01)$. Pacientes com alto escore nas escalas apresentaram maior prejuízo nas atividades rotineiras em relação aos pacientes sem desvio.

Quanto à área referente a relações pessoais, foi observada diferença estatisticamente significativa

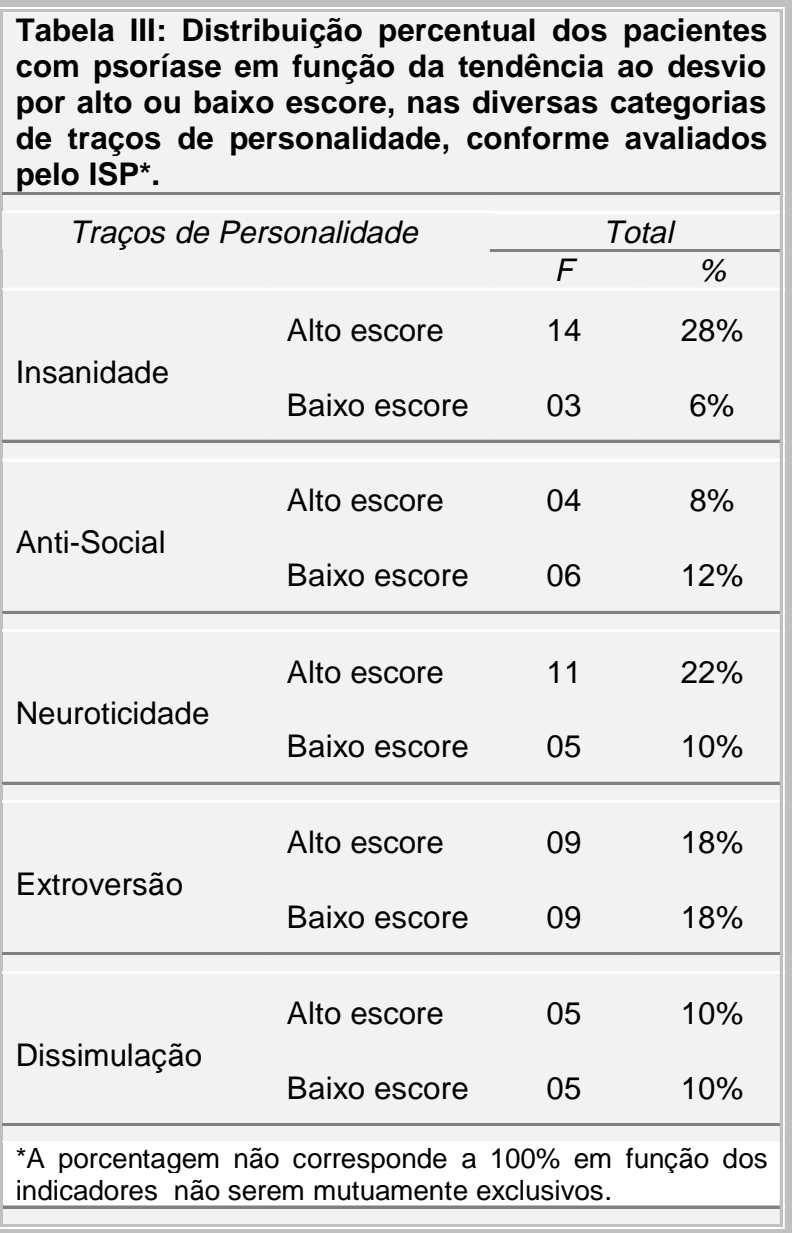




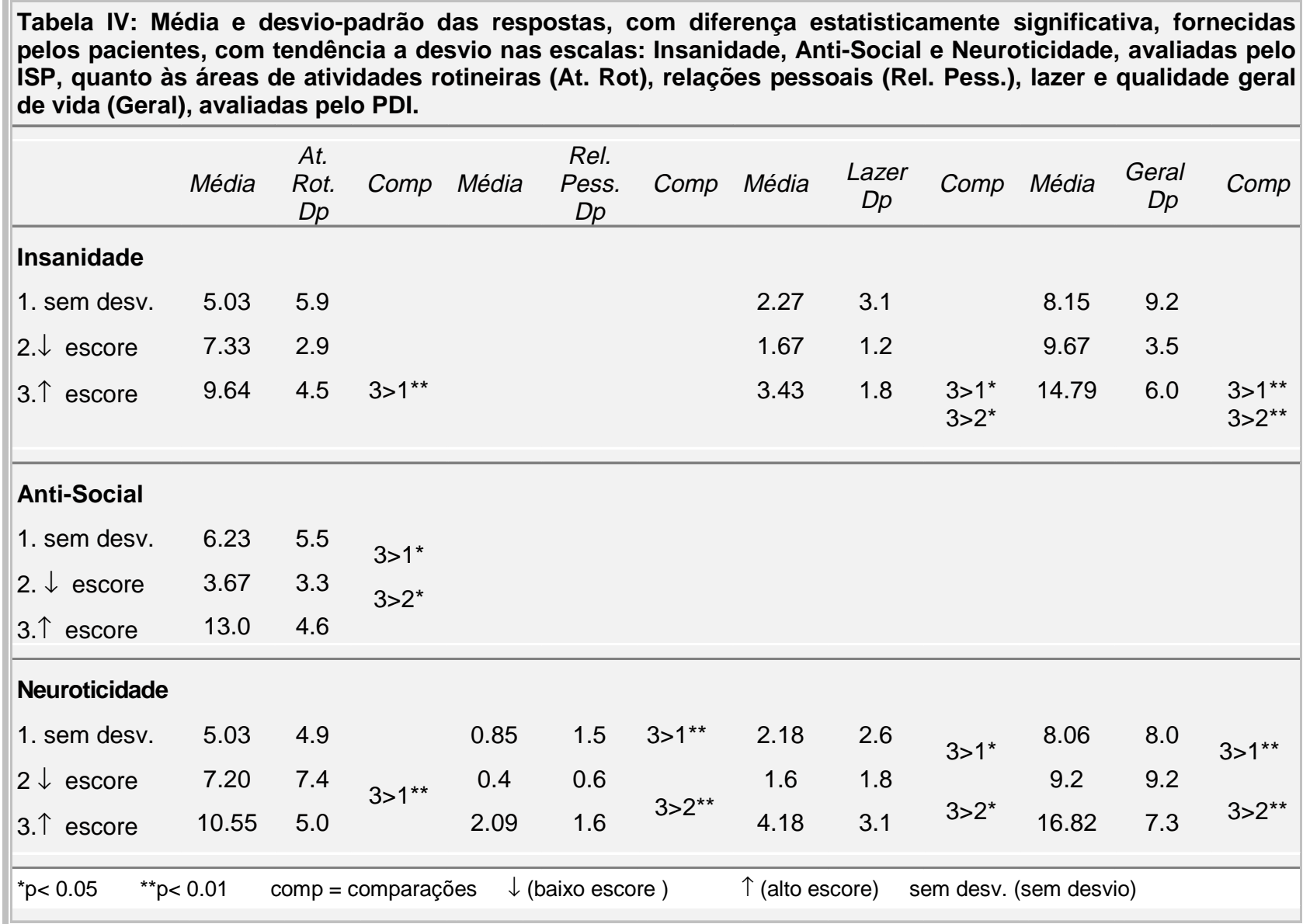

apenas na escala Neuroticidade. O grupo de pacientes com alto escore apresentou uma dificuldade, significativamente maior, nos relacionamentos interpessoais, em comparação ao grupo com baixo escore e sem desvio $(\mathrm{p}<0.01)$.

Com relação à área de lazer e qualidade geral de vida, os pacientes com alto escore de Insanidade apresentaram um prejuízo significativamente maior, em comparação aos pacientes sem desvio $(\mathrm{p}<0.01)$ e com baixo escore $(p<0.01)$. Observou-se a mesma tendência entre os pacientes com desvio de Neuroticidade. $\mathrm{O}$ grupo de pacientes com alto escore apresentou um prejuízo significativamente maior nesta área e em sua adaptação como um todo, em comparação ao grupo com baixo escore e sem desvio $(p<0.01)$ e $(p<0.01)$, respectivamente.

Na Tabela V, são apresentadas as atividades de maior prejuízo, relativas à área de atividades rotineiras, avaliadas pelo PDI, quando da presença de desvios na escala Insanidade, (ISP), através do teste de Kuskall-Wallys.
$\mathrm{Na}$ análise comparativa entre desvio de Insanidade e prejuízo em atividades específicas, observou-se que o grupo de pacientes com alto escore referiu, significativamente, maiores problemas que pacientes sem desvio relativamente a: usar serviços de cabeleireiro $(\mathrm{p}<0.001), \mathrm{b})$ aumentar a freqüência de banhos e repetitiva e freqüentemente lavar as áreas afetadas $(\mathrm{p}<0.01) \mathrm{c})$ ter problemas profissionais, como perda de emprego ou não admissão $(\mathrm{p}<0.01)$, sendo que, neste item, os pacientes com alto escore também apresentaram um prejuízo significativamente maior em relação aos com baixo escore $(\mathrm{p}<0.01)$.

Dentre as áreas específicas, avaliadas no PDI, as relativas a atividades rotineiras, relações pessoais e lazer apresentaram alguns itens mais susceptíveis à influência de desvios na escala de Neuroticidade, através da comparação no teste de Kuskall-Wallys, sendo os dados apresentados na Tabela VI.

Pacientes que apresentaram alto escore de Neuroticidade apresentam um prejuízo significativamente maior, em comparação aos pacientes sem des- 


\begin{tabular}{|c|c|c|c|c|c|c|c|c|c|}
\hline \multirow[t]{2}{*}{ Insanidade } & \multicolumn{3}{|c|}{ Problemas no cabeleireiro } & \multicolumn{3}{|c|}{ Aumento de banhos } & \multicolumn{3}{|c|}{ Dificuldades profissionais } \\
\hline & Média & $D p$ & Comp & Média & $D p$ & Comp & Média & $D p$ & Comp \\
\hline 1. Sem desvio & 0.48 & 0.9 & & 0.40 & 0.6 & & 0.58 & 1.0 & \\
\hline 2. $\downarrow$ escore & 1.0 & 1.0 & & 1.33 & 1.2 & & 0.33 & 0.6 & \\
\hline $3 . \uparrow$ escore & 1.64 & 1.0 & $3>1^{\star \star *}$ & 1.71 & 1.0 & $3>1^{* *}$ & 1.64 & 1.2 & $\begin{array}{l}3>1^{* *} \\
3>2^{* *}\end{array}$ \\
\hline${ }^{* \star} \mathrm{k}<0.01$ & & & ações & & & & & & \\
\hline
\end{tabular}

\begin{tabular}{|c|c|c|c|c|c|c|c|c|c|c|c|c|}
\hline \multirow[t]{2}{*}{ Neuroticidade } & \multicolumn{3}{|c|}{$\begin{array}{c}\text { Mudança na } \\
\text { realização de } \\
\text { atividades rotineiras }\end{array}$} & \multicolumn{3}{|c|}{$\begin{array}{l}\text { Dificuldades } \\
\text { profissionais }\end{array}$} & \multicolumn{3}{|c|}{ Dificuldades sexuais } & \multicolumn{3}{|c|}{$\begin{array}{c}\text { Dificuldades em } \\
\text { sair de casa }\end{array}$} \\
\hline & Média & $D p$ & Comp & Média & $D p$ & Comp & Média & $D p$ & Comp & Média & $D p$ & Comp \\
\hline 1. Sem desvio & 0.63 & 1.2 & & 0.50 & 0.9 & & 0.44 & 0.9 & & 0.62 & 0.9 & \\
\hline 2. $\downarrow$ escore & 0.75 & 1.5 & & 1.40 & 1.3 & & 0.20 & 0.5 & & 0 & 0 & \\
\hline \multirow[t]{2}{*}{$3 . \uparrow$ escore } & 1.67 & 0.8 & $3>1 *$ & 1.73 & 1.3 & $2>1 * *$ & 1.36 & 1.3 & $3>1 *$ & 1.08 & 1.2 & $3>1 *$ \\
\hline & & & $3>2 *$ & & & $3>1 * *$ & & & $3>2 *$ & & & $3>2 *$ \\
\hline${ }^{*} p<0.05$ & $p<0.01$ & & comp $=$ & omparaç & & & & & & & & \\
\hline
\end{tabular}

vio e com baixo escore, em atividades específicas, avaliadas nas áreas de atividades rotineiras, relações pessoais e lazer, como: a) mudanças na forma de desenvolvimento de suas atividades cotidianas $(\mathrm{p}<0.05)$, b) dificuldades sexuais $(\mathrm{p}<0.05), \mathrm{c})$ dificuldades em sair de casa $(\mathrm{p}<0.05)$.

Observou-se diferença quanto ao setor profissional, sendo que os pacientes com alto escore e baixo escore apresentaram dificuldades significativamente maiores nessa área, como perda de emprego ou não admissão, quando comparados aos pacientes sem desvio $(\mathrm{p}<0.01)$ e $(\mathrm{p}<0.01)$.

Na Tabela VII, são apresentados os dados relativos à comparação, através do teste de MannWhitney, entre insatisfação quanto à aparência física (entrevista) e impacto da psoríase nas atividades rotineiras e qualidade geral de vida (PDI).
O grupo de pacientes que relatou, na entrevista, insatisfação quanto à aparência física, apresentou indícios de prejuízo, significativamente maiores, nas atividades rotineiras e na qualidade geral de vida, quando comparado ao grupo com percepção satisfatória $(p<0.05)$ e $(p<0.01)$. Quando da comparação entre a percepção de melhora ou piora da lesão, relatada nas entrevistas, e a presença de prejuízo nas atividades rotineiras e qualidade geral de vida, não foram observadas diferenças estatisticamente significativas.

\section{DISCUSSÃO}

As áreas de atividades rotineiras e lazer, envolvendo principalmente atividades de higiene pessoal, uso de diferentes tipos de roupas, aumento na frequiência de banhos e troca de roupas, problemas no trabalho e 


\begin{tabular}{|c|c|c|c|c|c|}
\hline Áreas do $F$ & I X Aparência física & Média & Desvio- & Mediana & Comparações \\
\hline Atividades rotineiras & 1. Aparência satisfatória & 5.64 & 5.8 & 4 & $2>1^{*}$ \\
\hline & 2. Aparência insatisfatória & 8.34 & 5.5 & 7 & \\
\hline Qualidade geral de vida & 1. Aparência satisfatória & 8.24 & 8.7 & 5 & $2>1^{* *}$ \\
\hline & 2. Aparência insatisfatória & 13.54 & 8.5 & 13 & \\
\hline${ }^{*} p<0.0{ }^{* *} p<0.01$ & & & & & \\
\hline
\end{tabular}

na prática esportiva, têm sido indicadas como as mais vulneráveis ao impacto da psoríase na adaptação psicossocial, avaliadas através do Psoriasis Disability Index (PDI) ${ }^{(1,2,3)}$. Os pacientes avaliados no presente trabalho também apresentaram prejuízos adaptativos semelhantes aos mostrados nos estudos relatados, apesar da diferença de contexto sociocultural.

A extensão das lesões e a idade do paciente mostraram influenciar negativamente na qualidade de vida. Pacientes com lesões disseminadas indicaram maior prejuízo nas atividades rotineiras, lazer e qualidade geral de vida. Tais dados vão ao encontro dos resultados obtidos em estudo realizado com a utilização do PASI, para avaliação do grau de acometimento das lesões e um questionário para a análise do impacto psicossocial da psoríase ${ }^{(8)}$, onde se observou maior desadaptação psicossocial entre os pacientes com quadros clínicos mais graves. Manifestações disseminadas por todo o corpo fazem parte de quadros mais graves de psoríase, ocasionando maior limitação, em função de dificuldades físicas, além de produzir maior desfiguração na aparência física, o que favoreceu a discriminação. $\mathrm{O}$ grau de acometimento das lesões apresenta-se como uma variável relevante em se tratando de pacientes com psoríase, pois, além de prejuízos psicossociais, como os apontados anteriormente, um estudo realizado com esse grupo indicou manifestações psíquicas de depressão, associadas à presença exacerbada de lesões disseminadas ${ }^{(6)}$. Pacientes com idade entre 20-30 anos também apresentaram indícios de maior prejuízo nas relações pessoais, possivelmente por tratar-se de faixa etária que tem por característica maior inserção na rede social, o que expõe o jovem a diversos contatos e situações podem favorecer a discriminação. Problemas associados à aparência física e socialização também foi relatado em estudo com pacientes com psoríase, e o impacto da doença apresentou-se mais intenso na faixa etária de 18 a $45 \operatorname{anos}^{(4)}$.

Com relação aos aspectos psicológicos, a maioria dos pacientes apresentou desvios de personalidade em um ou mais traços de personalidade. Destacaram-se as escalas Extroversão, Insanidade e Neuroticidade, indicativas de dificuldades emocionais, compreendendo dificuldades no controle emocional e exibicionismo (Extroversão), manifestações psíquicas associadas a sentimentos de isolamento, ansiedade paranóide, e indicadores de possíveis distúrbios psiquiátricos (Insanidade), rigidez e alta suscetibilidade a ansiedade generalizada (Neuroticidade). Tais indícios sugerem a possibilidade de problemas psíquicos, dificultando a elaboração das vivências internas e externas, o que se reflete, possivelmente, em dificuldades adaptativas.

Problemas no funcionamento mental, favorecendo manifestações psicológicas, têm sido evidenciados em diversas pesquisas com pacientes com psoríase $^{(6,9,14)}$.Tais estudos constataram a presença de indicadores de ansiedade, impulsividade, defensividade, depressão, agressividade, e, ainda, dificuldades de simbolização e problemas referentes ao teste de realidade.

No presente estudo, indícios de problemas psicológicos apresentaram-se associados a maiores dificuldades em lidar com o impacto da doença na qualidade de vida. 
Indicadores de desvios de Insanidade, Neuroticidade e Anti-Social (ISP) mostraram-se associados a maior desadaptação psicossocial. Pacientes com desvio de Insanidade apresentaram maior prejuízo em suas atividades rotineiras, lazer e qualidade geral de vida, compreendendo principalmente o aumento na freqüência de banhos; problemas com cabeleireiro e dificuldades profissionais, como perda de emprego ou não admissão. Pacientes com desvio Anti-Social apresentaram maior prejuízo nas atividades rotineiras. Os pacientes que apresentaram desvio de Neuroticidade indicaram prejuízo em todos os setores avaliados, atividades rotineiras, relações pessoais, lazer e qualidade geral de vida, destacando-se os problemas profissionais, como perda de emprego, necessidade de mudar a forma de desenvolver as atividades rotineiras, em decorrência de limitações físicas e ainda problemas frente à sexualidade e dificuldade em sair de casa.

Com relação especificamente a prejuízos na esfera sexual, pacientes com psoríase têm apresentado um declínio na atividade sexual, associando tal fato ao constrangimento quanto à aparência física ${ }^{(15)}$. Quanto à sexualidade, sentimentos de exclusão e discriminação apresentaram-se exacerbados entre pacientes que apresentam lesões próximas aos genitais ou baixo ventre, em comparação aos pacientes com lesões em outras regiões do corpo ${ }^{(16)}$.

$\mathrm{Na}$ comparação entre indicadores de problemas de personalidade e suscetibilidade ao impacto da doença em atividades cotidianas específicas, observaram-se peculiaridades em subgrupos de pacientes quanto aos setores da adaptação social cujo componente psicológico mostrou-se proeminente. Apenas o subgrupo de pacientes com desvio de Neuroticidade apresentou restrição ao âmbito familiar, sugerindo dificuldades na socialização e problemas com a sexualidade. Problemas práticos, como dificuldades no trabalho, aumento na frequiência de banhos, foram relatados, principalmente, por pacientes com desvio de Insanidade.

Tal dado sugere a possível influência de indicadores psicopatológicos na adaptação psicossocial. Pacientes com maior comprometimento psicológico poderiam apresentar um maior distanciamento de si mesmos e de suas necessidades, tendo suas vivências pautadas pela satisfação imediata dos desejos, com certo distanciamento da avaliação externa. Assim, poderse-ia pensar que os pacientes com desvio de Insanidade, estariam mais voltados para a realização de suas necessidades práticas e menos suscetíveis à reação externa, o que lhes garantiria menor influência nas suas relações pessoais. Por outro lado, pacientes com desvio de Neuroticidade, por estarem mais voltados para si e com possibilidade de maior reflexão frente a suas vivências, poderiam sentir-se mais vulneráveis à reação externa, favorecendo maior prejuízo em suas relações pessoais. No caso dos pacientes com desvio Anti-Social, as características peculiares a tal traço, como o não reconhecimento da avaliação externa e da imposição de limites, podem ter favorecido uma menor fragilidade frente à reação externa e possível rejeição social, sendo percebido o impacto da doença apenas em atividades de ordem prática, ou seja, nas atividades rotineiras.

Estudos têm focalizado a importância da saúde mental e condições emocionais do paciente com psoríase na sua adaptação psicossocial, aderência e melhores resultados frente ao tratamento ${ }^{(6,17)}$. Pacientes com possibilidade de expressar suas vivências emocionais e angústias apresentaram menor número de prescrições médicas, indicando menor necessidade de diversas medicações visando à melhoria do quadro clínico $^{(6)}$. O suporte psicológico, como forma de auxiliar na vivência da psoríase, tem favorecido a melhor adaptação do paciente ao cotidiano ${ }^{(17)}$.

Avaliando-se, através da entrevista, a percepção dos pacientes frente às dificuldades associadas à doença e sua adaptação ao cotidiano, não se observaram relações entre prejuízo na área de atividades rotineiras e percepção de piora ou melhora das lesões.

No entanto, a percepção frente à aparência física mostrou-se associada a um maior prejuízo nas atividades cotidianas e desadaptação social. Os pacientes insatisfeitos quanto à aparência física referiram maior prejuízo nas atividades rotineiras e na qualidade geral de vida. Neste caso, a satisfação quanto a si próprio e imagem corporal parecem exercer influência maior, na adaptação psicossocial do paciente, do que a melhora concreta da lesão.

A importância de aspectos psicológicos na autoimagem e constituição da imagem corporal é destacada na literatura ${ }^{(18)}$, que pontua ser a imagem corporal, e consequientemente a satisfação quanto à aparência física, mais influenciada pelas condições emocionais que por características físicas concretas. No caso de pacientes com psoríase, tal concepção é reforçada em estudo $^{(10)}$ que constatou, no grupo, um maior grau de desadaptação social, associado à percepção de gravidade da doença que à realidade concreta da mesma. 
No presente trabalho, pode-se concluir que, além das características clínicas da psoríase, as condições psicológicas e satisfação quanto à aparência física são fatores que interferem no impacto da doença sobre a qualidade de vida. Tais dados apontam para as pecu- liaridades clínicas da doença e do funcionamento psicológico, influenciando a adaptação psicossocial, e sugerindo a importância do suporte psicológico como elemento complementar no atendimento a pacientes com psoríase.

MINGORANCE RC; LOUREIRO SR; OKINO L \& FOSS NT. Patients with psoriasis: psychosocial adaptation and personality features. Medicina, Ribeirão Preto, 34: 315-324, july/dec. 2001.

ABSTRACT: There has been increasing interest in the investigation of the psychological and social aspects of patients with psoriasis. The objective of the present study was to evaluate the psychological and adaptive conditions of patients with psoriasis and their relations with demographic variables and clinical condition. Twenty-five women and 25 men aged 20-50 years with a diagnosis of vulgar psoriasis were evaluated at the University Hospital, FMRP-USP. The Simplified Personality Inventory (SPI) and the Quality of Life Inventory (QLI) were applied in individual sessions according to technical recommendations and complemented with a semi-structured interview. The protocols were examined and compared to the normative data and the data were quantified and analyzed statistically. Eighty percent of the patients were found to present a tendency to personality deviation, with a predominance of the Extroversion, Insanity and Neuroticism scales. Most patients were in the 20 to 30 year age range and reported difficulties in psychosocial adaptation, especially in areas of routine activities $(\mathrm{p}<0.001)$, psoriatic extension, dissatisfaction about their physical appearance and Insanity and Neuroticism deviations, in association with impairment of routine activities, personal relations and leisure. The data indicate an inter-relation between psychological aspects, psychosocial adaptation and the clinical peculiarities of the disease.

UNITERMS: Psoriasis. Quality of Life. Psychology. Personality Inventory.

\section{REFERÊNCIAS BIBLIOGRÁFICAS}

1 - FINLAY AY; KHAN GK; LUSCOMBE DK \& SALEK MS. Validation of sickness impact profile and psoriasis disability index in psoriasis. Br J Dermatol 123: 751-756, 1990.

2 - KENT G \& AL'ABADIE M. The Psoriasis disability index - further analyses. Clin Exp Dermatol 18: 414- 416, 1993.

3 - FINLAY AY \& COLES EC. The affect of severe psoriasis on the quality of life of 369 patients. Br J Dermatol 132: 236244, 1995.

4 - GUPTA MA \& GUPTAAK. Age and gender differences in the impact of psoriasis on quality of life. Int J Dermatol 34: 700703, 1995.

5 - SCHMID-OT A; JAEGER B; KUENSEBECK HW; OTT R \& LAMPRECHT F. Dimensions of stigmatization in patients with psoriasis in a "Questionnarie on Experience with Skin Complaints". Dermatology 193: 304-310, 1996.

6 - SCHARLOO M, KAPTEIN A A, WEINMAN J, BERGMAN W, VERMEER BJ, \& ROOIJMANS HGM. Patients' illness perceptions and coping as predictors of functional status in psoriais: a 1-year follow-up. Br J Dermatol 142: 899-907, 2000.
7 - LUNDBERG L, JOHANNESSON M, SILVERDAHL M, HERMANSSON C \& LINDBERG M. Quality of life, health-state utilities and willingness to pay in patients with psoriasis and atopic eczema. Br J Dermatol 141: 1067-75, 1999.

8 - PERROT SB, MURRAY AH, LOWE J \& MATHIESON CM. The psychosocial impact of psoriasis: physical severity, quality of life, and stigmatization. Physiol Behav 70: 567$571,2000$.

9 - RUBINO IA; SONNINO A; PEZZAROSSA NC \& BASSI R. Personality disorders and psychiatric symptoms in psoriasis. Psychol Rep 77: 547-553, 1995.

10 - ROOT S; KENT G \& AL'ABADIE MSK. The relationship between disease severity, disability and psychological distress in patients undergoing PUVA tratment for psoriasis. Dermatology 189: 234-237, 1994.

11 - LANYON RI. Development and validation of a psychological screening invetory. J Consult Clin Psychol 35: 1-24, 1970.

12 - CABRERA CC. Comparação dos resultados obtidos pela aplicação de um teste em portadores de úlcera péptica duodenal com os resultados obtidos em uma população de referência. Dissertação de Mestrado, Faculdade de Medicina de Ribeirão Preto da USP. Departamento de Neurologia, Psiquiatria e Psicologia Médica, Ribeirão Preto, p.118, 1991. 
13 - FINLAY AY \& KELLY SE. Psoriasis - an index of disability. Clin Exp Dermatol 12: 8-11, 1987.

14 - ANDREOLI E; BONDAFOGLIO PG; MAZZETTI M; MOZZETTA A; NICOLAI P; PUDDU P \& DECAMINADA F. Characteristics and possible significance of the answers to Rorschach from patients suffering from psoriasis. Acta Derm Venereol 186: 154-157, 1994. Suppl.

15 - GUPTA MA \& GUPTA AK. Psoriasis and sex: A Study of moderately to severely affected patients. Int $\mathbf{J}$ Dermatol 36: 259-262, 1997.

16 - SCHMID-OTT G, KUENSEBECK H-W, BURKARD J, WERFEL T, FRAHM K, RUITMAN J, KAPP A \& LAMPRECHT F. Vality study for the stigmatization experience in atopic dermatitis and psoriatic patients. Acta Derm Venereol. 79: 443-447, 1999.
17 - SENG TK \& NEE TS. Group therapy: a useful and supportive treatment for psoriasis patients. Int J Dermatol 36: 110-112, 1997.

18 - CAPISANO HF. Imagem corporal. In: MELLO FILHO, J, ed. Psicossomática hoje, Artes Médicas, Porto Alegre, p. 179192, 1992.

Recebido para publicação em 19/03/2001

Aprovado para publicação em 02/08/2001 